\title{
Diagnostic dilemma in managing relapsing episodes of Cryptococcal meningitis in HIV infected patient; a case report
}

\author{
Pannala. W.S ${ }^{1}$, Godakandaarachchi. L.P.P $P^{2}$, Wijewickrama. $A^{3}$, Ranatunga. J. $D^{4}$
}

\begin{abstract}
HIV associated morbidity and mortality has dramatically been reduced over the past decade with introduction of antiretroviral therapy (ART). However, significant number of undiagnosed patients still present with advanced HIV disease. Cryptococcal meningitis, commonly caused by the fungus Cryptococcus neoformans, is one of the commonest central nervous system opportunistic infection among those who diagnosed during advanced stage of disease. Relapsing episodes of Cryptococcal meningitis is not uncommon following successful treatment. Relapses may occur due to treatment failure or development of paradoxical cryptococcal immune reconstitution inflammatory response (IRIS) following immunological recovery with ART. However, the exact cause of relapse occurring after commencement of ART is indistinguishable from treatment failures and IRIS.

Here, we present a case of a patient with HIV who had been treated for Cryptococcal meningitis that subsequently developed two recurrent episodes of Cryptococcal meningitis after initial Cryptococcal treatment, within consecutive two months while on ART.
\end{abstract}

Key words: HIV, ART, Immune Reconstitution Inflammatory Syndrome, IRIS, Paradoxical Cryptococcal IRIS

Authors: corresponding author; ${ }^{1}$ Dr W.S.Pannala, MBBS, PgD Ven; Registrar, National STD/AIDS Control Programme, No 29, De Saram Place, Colombo 10, Sri Lanka. Email: warunip@yahoo.com

${ }^{2}$ Dr L.P.P Godakandaarachchi, MBBS, PgD Ven; Registrar, North Colombo Teaching Hospital, Ragama, Sri

Lanka,Email:piyumika8@gmail.com

${ }^{3}$ Dr.A.Wijewickrama,MBBS, MD, MRCP (UK); consultant physician, National Institute of Infectious Diseases, Angoda, Sri Lanka

${ }^{4}$ Dr.J.D.Ranatunga, MBBS, PgD Ven,MD; consultant venereologist, North Colombo Teaching Hospital, Ragama, Sri Lanka

Acknowledgement: Professorial medical unit, NCTH, Ragama, Sri Lanka, staff of the NIID, Angada and STD clinic, Ragama, Sri Lanka

Conflict of interest: Authors claim no conflicts of interest

Funding: No funding support for this case report

Originality: This is an original work and there have not been any previous publications

Submitted: 09.11.2016, Accepted: 20.12.2016

\section{Full article}

\section{Introduction}

HIV associated morbidity and mortality has dramatically been reduced over the past with introduction of antiretroviral therapy (ART). However, late presentations of disease still remain to be a problem even in developed world. Cryptococcal meningitis is one of the commonest life threatening central nervous system opportunistic infection among patients with advanced HIV disease. Causative organism, Cryptococcus is encapsulated yeast, ubiquitous in the environment. The organisms most commonly associated with HIV-related cryptococcal disease are $C$. neoformans var. grubii, neoformans and gattii. Epidemiological studies have confirmed that primary infections occur during childhood and are usually asymptomatic (1). Infection occurs following inhalation of organism and result in localized disease in the lung. Conditions in which the host immune system is compromised, such as HIV infection, organism disseminates from the lung to other organs, especially to the brain, where it causes the meningoencephalitis (2).

According to the latest $\mathrm{CDC}$ recommendations (Centers for Disease Control and Prevention, USA) treatment of cryptococcal meningitis 
consists of three phases, Induction therapy, consolidation therapy and maintenance therapy. For induction, Amphotericin B formulations are given intravenously in combination with oral flucytosine. After two weeks of successful induction therapy consolidation therapy is initiated with fluconazole $400 \mathrm{mg}$ daily and continued at least 8 weeks. Subsequently fluconazole should be reduced to $200 \mathrm{mg}$ daily and continue as chronic maintenance therapy to complete at least one year. High dose flucanazole monotherapy (1200md daily) is inferior to amphotericin $B$, depending on the early fungicidal activity and can be given alternatively for induction therapy only for those who cannot tolerate amphotericin B.

However, subgroup of patients will develop relapsing episodes of meningitis after successful initial response. This can either be due to treatment failure or paradoxical cryptococcal IRIS.

Treatment failure is defined as "a lack of clinical improvement and continued positive CSF cultures after 2 weeks of appropriate therapy, including management of increased intracranial pressure (ICP) or as a relapse after an initial clinical response, defined as recurrence of symptoms with a positive CSF culture after $\geq 4$ weeks of treatment"(3). Whereas in IRIS patients experiences a clinical deterioration as a consequence of rapid and dysregulated restoration of antigen specific immune responses during the treatment with antiretroviral therapy or ART(4). Although there is no gold standard definition for IRIS, it is generally accepted that certain minimum requirements should be fulfilled to diagnose IRIS. A temporal association between ART initiation and the subsequent worsening of symptoms, evidence of immune restoration with ART and exclusion of possible other pathologies are among them(4).

Paradoxical Cryptococcal-IRIS occurs around $6 \%$ to $45 \%$ patients with HIV associated Cryptococcal meningitis who survive to start ART(5). However, Cryptococcal-IRIS is usually a diagnosis of exclusion, making it a diagnostic dilemma especially in resource limited settings. Other than several case reports and few retrospective and prospective studies published in other countries, to our knowledge there are no published materials in Sri Lanka in this regard.

Here, we present a case of a newly diagnosed patient with HIV who had been treated for Cryptococcal meningitis that subsequently developed two recurrent episodes of meningitis after initial treatment response, within consecutive two months while on antiretroviral therapy.

\section{Case history}

28 year old married male presented at North Colombo Teaching Hospital (NCTH), Sri Lanka on first week of May 2016 with a history of intermittent fever and headache associated with loss of appetite and loss of weight for last three months duration. At onset, the fever was low grade. However, during the later part of the illness, he developed high grade fever with chills which needed hospital admission. He was investigated and treated as an outpatient in private sector for several weeks when he admitted to NCTH. Though he complained on and off dry cough, he did not have haemoptysis or significant shortness of breath on exertion. He had left sided moderate headache without photophobia, vomiting or limb weakness. He neither had altered level of conciseness nor the seizures. The patient was investigated at NCTH as a case of pyrexia of unknown origin (PUO) and found to have positive HIV screening test which was confirmed by the Western blot test. His baseline liver and renal functions were within normal limits. He had moderate normochromic normocytic anaemia $(\mathrm{Hb}$ $9.8 \mathrm{~g} / \mathrm{dl}$ ) which was reported as anaemia of chronic disease in blood picture. Pulmonary TB was excluded by negative mantoux test, chest radiograph and three negative sputum samples for Acid-fast Bcilli (AFB). Sputum was also negative for Tuberculosis culture as well as GeneXpert MTB assay. He was negative for 
Hepatitis B surface antigen, Hepatitis C antibody and toxoplasma antibodies. However, cytomegalovirus (CMV) antibody came as positive, but CMV was not detected by DNA PCR. He did not have focal lesions or cerebral oedema on contrast-enhanced computed tomography (CECT) brain.

The lumber puncture revealed no CSF pleocytosis, slightly elevated CSF protein $(54.6 \mathrm{mg} / \mathrm{dl})$ and reduced glucose levels $(31 \mathrm{mg} / \mathrm{dl})$. C. neoformans was positive in India Ink preparations and cryptococcal cultures along with positive serum and CSF cryptococcal antigens, confirmed the diagnosis. Furthermore, CSF was negative for bacterial cultures, Tuberculosis cultures as well as GeneXpert MTB. His baseline CD4 count was 78 cells $/ \mu$ l and baseline HIV-1 viral load was 920,487 copies/ml.

Around third week of admission, the patient was commenced with amphotericin B deoxycholate $0.7 \mathrm{mg} / \mathrm{kg}$ IV daily for induction phase of the cryptococcal meningitis treatment. However, he was unable to receive treatment for more than 5 days due to rising serum creatinine levels. (from $79 \mu \mathrm{mol} / \mathrm{l}$ to $174 \mu \mathrm{mol} / \mathrm{l})$. Thereafter, he was treated with oral fluconazole $1,200 \mathrm{mg}$ daily for two weeks (14 days) as the induction phase of treatment. The patient's serum creatinine was reduced back to normal levels after discontinuation of treatment with amphotericin B deoxycholate. At the completion of 2 weeks of induction therapy fluconazole dose was reduced to $400 \mathrm{mg}$ once daily treatment which was planned to be given in next 8 weeks as the consolidation phase of the treatment. After completion of two weeks induction phase, the patient was symptom free. However, microbiological response was not assessed with repeat CSF cultures. Around the third week of cryptococcal meningitis therapy, ART was started with tenofovir, emtricitabine and efavirenz (TDF+FTC+EFV) regimen. The patient well tolerated ART and was asymptomatic thereafter, until completion of two weeks of ART when he again developed moderately severe on and off headache without any fever or vomiting. But the patient continued to have ART and fluconazole without seeking medical advice for the next four weeks.

Then he admitted at infectious disease hospital (IDH), Angoda on the first week of August 2016 with one episode of altered consciousness which persisted about 2 hours on previous day. On admission he was on ART and fluconazole $400 \mathrm{mg}$ daily for last six week. He was having moderately severe generalized headache for about last four weeks without fever, vomiting or neurological weakness. He was also having good compliance to both drugs on admission. The patient was conscious, rational and afebrile and had neither features of meningeal irritation nor focal neurological signs at presentation. His respiratory, cardiovascular and abdominal examinations were normal. His renal functions, liver functions and haematological parameters were within normal limits at this admission.

The lumber puncture at IDH revealed marked CSF inflammation with a WBC count of 27 cell $/ \mu \mathrm{l}$ (100\% lymphocytes), $102 \mathrm{mg} / \mathrm{dl}$ protein. CSF was positive for cryptococcal antigens, whereas CSF India ink stain and cryptococcal culture became negative. High pressure flow of CSF through the needle was observed during the lumber puncture, but the exact CSF pressure was not measured. CSF cultures for bacteria and mycobacteria were also negative and the computed tomography (CT) of the brain did not show any focal lesions. While waiting for culture results, empirical therapy was started with high dose cefotaxime and liposomal amphotericin B $3 \mathrm{mg} / \mathrm{kg}$ IV daily and ART was continued without interruption. The patient's headache was fully resolved with this treatment together with serial lumber punctures in first two consecutive days of admission with removal of $20-30 \mathrm{ml}$ of CSF during each time. He only experienced marginal serum creatinine rise with liposomal amphotericin B, comparing with initial Amphotericin B deoxycholate therapy. During the hospital stay patient did not experience any new symptoms or signs and two weeks of 
induction period of liposomal amphotericin B was successfully completed.

The patient was discharged on the $20^{\text {th }}$ day, few days after restarting fluconazole $400 \mathrm{mg}$ daily as consolidated phase of cryptococcal therapy and remained asymptomatic while receiving ART and fluconazole until he readmitted after 4 days of discharge with reappearance of headache, severe vomiting and one episode of self limited left sided body weakness. On admission patient was conscious, rational and afebrile but having positive neck stiffness and Kernig's signs indicating meningeal irritation. But he did not have any focal neurological signs. Noncontrast computed tomography (NCCT) of the brain was normal on admission. CSF revealed $72 \mathrm{mg} / \mathrm{dl}$ protein, low sugar and 14 cells $/ \mu \mathrm{l}$ (100\% lymphocytes). CSF was still positive for Cryptococcal antigen and the India ink stain and Cryptococcal culture were negative. During the lumbar puncture procedures (sitting position), high pressure flow of CSF through the needle was observed, but the exact CSF pressure was not measured. Patient's symptoms were resolved with another two serial lumber on two consecutive days removing $30 \mathrm{ml}$ of CSF during each time. The patient was continued with ART and fluconazole $400 \mathrm{mg}$ daily.

At the beginning of the first relapse, his CD4 count had been increased to 246 cells/ $\mu$ l from baseline value of 76 cells/ $\mu$ l and HIV1 viral load was reduced to 49 copies $/ \mathrm{ml}$ indicating rapid immunological and virological recovery with six weeks of ART.

Although the relapses were initially considered as treatment failures, considering negative fungal cultures as well as rapid immunological and virological response to ART, both relapses were assumed to be caused by paradoxical cryptococcal IRIS retrospectively. He received 8 weeks fluconazole $400 \mathrm{mg}$ daily as consolidation phase of cryptococcal therapy without interruption and currently fully recovered and in good condition with ART and fluconazole $200 \mathrm{mg}$ daily maintenance therapy.

\section{Discussion}

Serum cryptococcal antigen is almost always positive in patients with cryptococcal meningitis (3). All patients with a positive serum cryptococcal antigen should undergo further evaluation by lumbar puncture after CT or MRI cerebral scanning(1). A positive CSF cryptococcal antigen, Indian ink stain of CSF, or CSF Cryptococcus culture confirms meningitis(1). Up to $75 \%$ patients with cryptococcal meningitis have routine blood cultures positive for Cryptococcal neoformans (3). As our patient had positive serum and CSF cryptococcal antigen together with positive CSF India ink stain and positive CSF cryptococcal culture he fulfilled all the criteria to diagnose HIV associated cryptococcal meningitis during the first presentation at NCTH, Ragama. However, his blood cultures were not positive for Cryptococcal neoformans.

Paradoxical Cryptococcal IRIS occurs as worsening or recurring already treated Cryptococcal disease in the same or new anatomical site, despite microbiologic treatment success (6). Other than the commoner CNS manifestations like meningeal disease and cryptococcoma, C-IRIS can present as non-CNS manifestations like fever, eye disease, suppurating soft tissue lesions, hypercalcemia or cavitative and nodular pulmonary disease (6). Reported time to develop paradoxical C-IRIS after ART varies widely with median time ranging from 1 to 10 months (6).

In our case patient was clinically responded during initial two weeks of antifungal therapy and presented with worsening CNS symptoms at six weeks of ART. However, we were not certain about the patient's initial microbiological treatment success after treatment as we did not perform repeat lumber puncture and CSF cultures following completion of induction phase of therapy.

Risk factors for paradoxical-IRIS have been reported as high HIV viral load prior to ART, 
earlier initiation of ART after treating the disease, low initial CD4 count and greater CD4 recovery during first 6 months and presence of fungaemia with higher serum cryptococcal antigen titer at pre-ART (6). However, in some prospective cohorts, HIV-1 viral load, time to start ART, and baseline CD4 were not risk factors for C-IRI (6). A paucity of CSF inflammation at the time of initial Cryptococcal meningitis diagnosis has also been associated with subsequent development of IRIS (7).

As our patient was commenced with ART just after completion of two weeks induction therapy despite paucity of CSF inflammation, very high baseline HIV viral load and low baseline CD4 count, favour the diagnosis of paradoxical cryptococcal IRIS.

Although widely available CSF parameters like protein, WBC levels are informative of IRIS risk, particular cut point as an ideal diagnostic threshold has not been identified. But, those with more prominent CSF inflammation like WBC count $>25$ cells $/ \mu$ l and protein $>50$ $\mathrm{mg} / \mathrm{dl}$, at the time of cryptococcal diagnosis, infrequently develop IRIS once receiving ART. Therefore, high risk patients may be identifiable prior to ART according to case by case basis (7).

One prospective study done in Uganda found pre-ART increase in serum Th17 and Th2 responses (IL17 and IL4) and lack of pro inflammatory cytokine responses (TNF $\alpha$, granulocyte colony stimulating factor, vascular endothelial growth factor) predispose individuals to subsequent IRIS (8). After validation these biomarkers might be an objective tool to predict the risk of C-IRIS and guide the timing of ART.

Meningeal C-IRIS is usually indistinguishable from a relapse at the time of presentation. CSF culture results may help to differentiate the two clinical scenarios. However, negative CSF culture is not an absolute requirement to diagnose C-IRIS due to the variable time taken for CSF sterility after treating with recommended antifungal medications. But a positive fungal culture after three months of antifungal therapy can be considered as treatment failure and exclude the diagnosis of C-IRIS (9).

In our case patient was initially treated sub optimally with fluconazole monotherapy induction and he developed the first relapse at six weeks of ART and fluconazole therapy. Therefore, we were unable to exclude the diagnosis of treatment failure and justified the retreatment of patient with optimal induction therapy.

However, during the second relapse, considering the repeatedly negative Cryptococcal cultures, rapid immunological and virological recovery with ART as well as successful completion of liposomal amphotericin $B$ induction therapy during first relapse, the diagnosis of paradoxical cryptococcal IRIS was quiet certain. Two Serial lumber punctures with removal of $20-30 \mathrm{ml}$ of CSF at a time together with ART and fluconazole consolidation therapy alleviated the symptoms of the patient during this relapse.

The treatment failure of cryptococcal meningitis may be due to poor adherence to antifungal medications or fluconazole resistance. As primary resistance to fluconazole is uncommon for Cryptococcal neoformans, susceptibility testing is not routinely recommended for initial management of cryptococcosis (3). However, isolates collected during a relapse should be checked for drug susceptibility.

Optimal therapy for patients with treatment failure has not been established. According to CDC recommendations patients who fail to respond fluconazole monotherapy induction should be switched to amphotericin B, with or without flucytosine. "Those initially treated with an amphotericin B formulation should remain on it until a clinical response occurs. Liposomal amphotericin B or amphotericin B lipid complex is better tolerated and has 
greater efficacy than deoxycholate formulation in this setting and should be considered when initial treatment with other regimens fails"(3).

Timing of ART for cryptococcosis is a therapeutic dilemma due to conflicting data. Most available studies are also underpowered to provide definitive guidance (10). According to the CDC recommendation timing of ART administration should be considered between 2 and 10 weeks after the start of antifungal therapy with the precise starting dates based on individual conditions and local experience. Multisite randomized trial done in Uganda and South Africa showed deferred ART until 5 weeks after the start of amphotericin therapy had improved survival comparing with ART initiated at 1 to 2 weeks. The findings were especially significant, among the patients with a paucity of white cells in CSF (3).

Recommended management of Cryptococcal IRIS according to CDC guidelines are to continue both ART and antifungal therapy and reduction of CSF pressure if present. However, several trials have suggested steroids as well as non steroidal anti inflammatory drugs for the treatment of IRIS but none of them are clearly proven.

Considering available literature and our experience we also recommend, evaluation of each case should be done carefully and individually in case by case basis, based on available resources and expertise to decide on ART timing, diagnosis and the management of cryptococcal meningitis and its complications.

\section{References}

1. Lane FB, London N. British HIV Association guidelines for the treatment of opportunistic infection in HIV-positive individuals $2010 \mathrm{M}$ Nelson ,D Dockrell , S Edwards on behalf of the BHIVA
Guidelines See Appendix 2 for list of members of the BHIVA Guidelines Subcommittee Op. Infection. 2010;

2. Litvintseva AP, Mitchell TG. Population Genetic Analyses Reveal the African Origin and Strain Variation of Cryptococcus neoformans var . grubii. plos Pathog. 2012;8(2):8-11.

3. Centers for Disease Control and Prevention. Guidelines for the Prevention and Treatment of Opportunistic Infections in HIV-Infected Adults. 2014;149-60. Available from: https://aidsinfo.nih.gov/contentfiles/lvguidelines/a dult_oi.pdf

4. Sharma SK, Soneja M. HIV \& immune reconstitution inflammatory syndrome (IRIS). Indian J Med Res [Internet]. 2011;134(December):866-77. Available from:

http://www.pubmedcentral.nih.gov/articlerender.f cgi?artid=3284095\&tool=pmcentrez \&rendertype $=a$ bstract

5. Longley N, Harrison TS, Jarvis JN. Cryptococcal immune reconstitution inflammatory syndrome. Curr Opin Infect Dis. 2013;26(1):26-34.

6. Haddow LJ, Colebunders R, Meintjes G, Lawn SD, Elliott JH, Easterbrook PJ, et al. Cryptococcal Immune Reconstitution Inflammatory Syndrome in HIV-1-infected individuals: Literature Review and Proposed Clinical Case Definitions. Lancet Infect Dis. 2010;10(11):791-802.

7. Boulware DR, Bonham SC, Meya DB, Wiesner DL, Park GS, Kambugu A, et al. Paucity of initial cerebrospinal fluid inflammation in cryptococcal meningitis is associated with subsequent immune reconstitution inflammatory syndrome. J Infect Dis. 2010;202(6):962-70.

8. Boulware DR, Meya DB, Bergemann TL, Wiesner DL, Rhein J, Musubire A, et al. Clinical features and serum biomarkers in HIV immune reconstitution inflammatory syndrome after cryptococcal meningitis: A prospective cohort study. PLoS Med. 2010;7(12):1-14.

9. Nunnari G, Gussio M, Pinzone MR, Martellotta F, Cosentino S, Cacopardo B, et al. Cryptococcal meningitis in an HIV-1-infected person: Relapses or IRIS? Case report and review of the literature. Eur Rev Med Pharmacol Sci. 2013;17(11):1555-9.

10. Makadzange AT, Ndhlovu CE, Takarinda K, Reid M, Kurangwa $\mathrm{M}$, Gona $\mathrm{P}$, et al. Early versus Delayed Initiation of Antiretroviral Therapy for Concurrent HIV Infection and Cryptococcal Meningitis in Sub-Saharan Africa. Clin Infect Dis [Internet]. 2010;50(11):1532-8. Available from: http://cid.oxfordjournals.org/lookup/doi/10.1086/ 652652 